\title{
Nanomechanical Properties of Bordetella Pertussis Depend on Virulence and Neighborhood
}

\author{
M.E. Vela ${ }^{1 *}$, M.I. Villalba ${ }^{2}$, L.Venturelli ${ }^{3}$, G.Dietler ${ }^{3}$, O.M. Yantorno ${ }^{2}$, S. Kasas $^{3}$ \\ ${ }^{1}$ Laboratorio de Nanoscopías y Fisicoquímica de Superficies (INIFTA (UNLP-CONICET)) CC \\ 16. Suc. 4, 1900 La Plata, Argentina. \\ ${ }^{2}$ Centro de Investigación y Desarrollo de Fermentaciones Industriales (CINDEFI-CONICET- \\ CCT La Plata), Facultad de Ciencias Exactas, 1900 La Plata, Argentina \\ ${ }^{3}$ Laboratoire de Physique de la Matière Vivante, EPFL, 1015 Lausanne, Switzerland \\ mevela@inifta.unlp.edu.ar
}

Atomic force microscopy (AFM) is a widely used tool in microbiology to image and explore the nanomechanical properties of bacteria. ${ }^{1,2}$ In this work, we employed this technique to study the membrane properties of Bordetella pertussis, a Gram-negative bacterium that colonizes human airway and produces whooping cough. More specifically, we analyzed the effect of two antibiotics, erythromycin and ampicillin, on the bacterial envelope and monitored cell wall stiffness modifications during virulence changes.

Bacteria from over-night liquid culture were attached to a Petri dish using polylysine $0.01 \%$ and analyzed in liquid medium by AFM Quantitative Imaging mode. Data were processed using JPK data processing software and home-made programs written in MatLab R2013b. Elasticity and morphological results revealed a decrease in the membrane stiffness and in the height of $B$. pertussis after 5 hours of ampicillin and erythromycin exposure, which is in accordance with previous studies on other pathogenic bacteria. Interestingly we noticed that the stiffness drops after antibiotic exposure for bacteria being members of a group (i.e. in contact) in comparison with the stiffness of individual bacteria. We also could demonstrate that the stiffness of $B$. pertussis envelope reaches its maximum value during the process of transformation from avirulent to virulence phase. These results could be interpreted on the basis of the different mechanisms of both antibiotics on the bacteria where ampicillin inhibits peptidoglycan bridges and erythromycin restricts protein synthesis. We hope these findings will contribute to a better understanding of the mechanisms of pathogenesis and antibiotic resistance of $\mathrm{B}$. pertussis.

\section{References}

[1] S. Kasas et al., Journal of Physics D: Applied Physics 46(13) (2013), 133001

[2] L. Arnal et al. , Nanoscale 7(41), (2015), p. 17563 


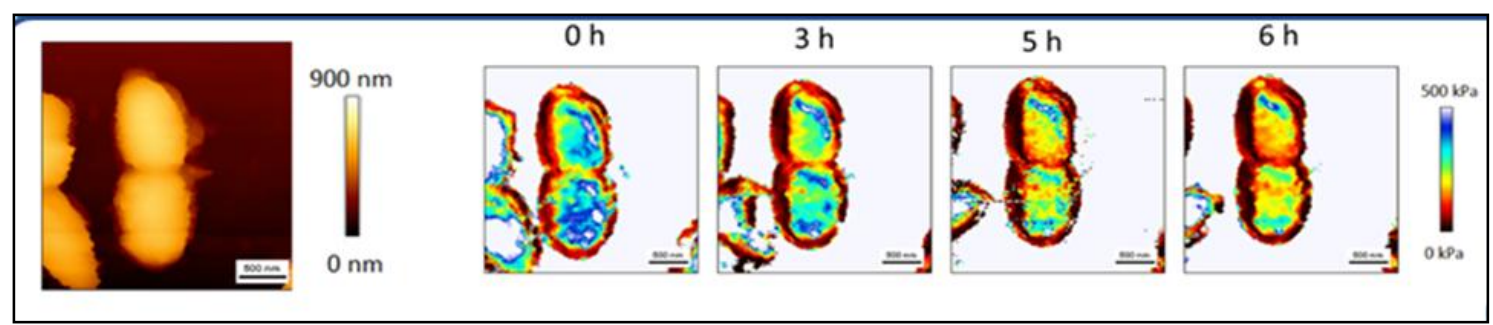

Topographic and stiffness images of Bordetella Pertussis bacteria during Erythromicin exposure 